\title{
Water masers in NLS1 galaxies
}

\author{
Andrea Tarchi* \\ INAF-Osservatorio Astronomico di Cagliari, Loc. Poggio dei Pini, Strada 54, I-09012 \\ Capoterra (CA), Italy \\ E-mail: atarchi@oa-cagliari.inaf.it
}

\section{Paola Castangia}

INAF-Osservatorio Astronomico di Cagliari, Loc. Poggio dei Pini, Strada 54, I-09012

Capoterra (CA), Italy

E-mail: pcastang@oa-cagliari.inaf.it

\section{Alberto Columbano}

INAF-Osservatorio Astronomico di Cagliari, Loc. Poggio dei Pini, Strada 54, I-09012

Capoterra (CA), Italy

E-mail: columban@oa-cagliari.inaf.it

\section{Francesca Panessa}

IASF/INAF, via del Fosso del Cavaliere 100, 00133 Roma, Italy

E-mail: francesca.panessadiasf-roma.inaf.it

\section{Jim A. Braatz}

National Radio Astronomy Observatory, 520 Edgemont Road, Charlottesville, VA 22903, USA

E-mail: jbraatz@nrao.edu

\begin{abstract}
Luminous extragalactic water masers originate in warm and dense gas in the innermost regions of AGN tracing circumnuclear accretion disks, relativistic jets, or nuclear outflows. So far, the majority of water maser sources have been detected in Sy2 galaxies. Among the few exceptions are the water masers hosted by Narrow-Line Seyfert 1 (NLS1) galaxies. We have studied available line and continuum data at radio frequencies of two NLS1 galaxies, NGC 4051 and NGC 5506, where water maser emission has been detected, to investigate the interplay between this peculiar class of AGN and the maser phenomenon. In this talk, we will illustrate the main results of this study in the framework of a possible association of the water maser sources in NLS1 with radio jets, outflows, or accretion disks.
\end{abstract}

Narrow-Line Seyfert 1 Galaxies and their place in the Universe - NLS1, April 04-06, 2011

Milan Italy

\footnotetext{
${ }^{*}$ Speaker.
} 


\section{Introduction}

Most extragalactic $\mathrm{H}_{2} \mathrm{O}$ masers are associated with AGN, where they have been related with three distinct phenomena. (1) They may form directly in the nuclear accretion disk, where they can be used to trace the disk geometry, rotation velocity, and where they may reveal the enclosed nuclear mass. In some cases they are also being used to measure accurate distances to their host galaxies (for the most recent case of UGC 3789, see [3]). (2) They are associated with radio jets, where they are either the result of an interaction between the jet(s) and an encroaching molecular cloud or due to an accidental overlap along the line-of-sight between a cloud and the radio continuum of the jet (NGC 1052: e. g. [17]; NGC 1068: e. g. [4]; Mrk 348: e. g. [14]). These masers can provide important information about the evolution of jets and their hotspots. (3) They are associated with nuclear outflows, tracing the velocity and geometry of nuclear winds at $<1 \mathrm{pc}$ from the nucleus, as in the case of Circinus ([6]).

So far, more than 3000 galaxies have been searched for water maser emission and detections have been obtained in about 130 of them (Braatz et al., in prep), the majority being Sy 2 galaxies (active galaxies with narrow emission line optical spectra). Very few have instead been found in type 1 Seyfert galaxies but, interestingly, most of them in host galaxies classified as Narrow Line Seyfert 1 (NLS1; Tarchi et al. submitted). This class of AGN has all properties of type 1 Seyfert galaxies but with the narrowest Balmer lines from the Broad Line Region and the strongest Fe II emission. Extreme properties are also observed in X-rays, such as a strong soft excess emission below $1 \mathrm{keV}$ and rapid flux variability, accompanied by steeper photon indices with respect to Seyfert 1 galaxies (see [11] for a review).

Presently, there are only two maser NLS1 galaxies with available interferometric measurements of both the radio continuum (with VLBI) and the maser emission (with the VLA): NGC 4051 and NGC 5506. These are the targets of the present work.

\section{Observations and data reduction}

We have retrieved archival VLA observations of the water maser line for both NGC 4051 and NGC 5506. For the former galaxy, A-array configuration data were available, while for the latter the observations were performed in both $\mathrm{DnC}$ and $\mathrm{A}$ configurations. We also incorporate broad-band VLA A-array 22-GHz continuum data of NGC 5506.

The VLA data were reduced and analyzed using AIPS. The VLA water maser spectrum of NGC 4051 has been displayed using CLASS, a package implemented in the GILDAS software.

\section{Results and preliminary conclusions}

NGC 4051: The nuclear radio continuum has been imaged by [5] at both 1.6 and $5 \mathrm{GHz}$ with the EVN. The nuclear radio source is most easily explained in terms of a jet-base/outflow structure. Indeed, an ejection process is suggested by the three aligned sub-millijansky components detected by [5] in NGC 4051. The position of the $\mathrm{H}_{2} \mathrm{O}$ maser derived by [8] and ourselves is coincident, within an accuracy of 0.1 arcsec (corresponding to $5 \mathrm{pc}$ at the distance of NGC 4051, with the EVN radio core. This may suggest that the water maser emission arises from a molecular disk or from 


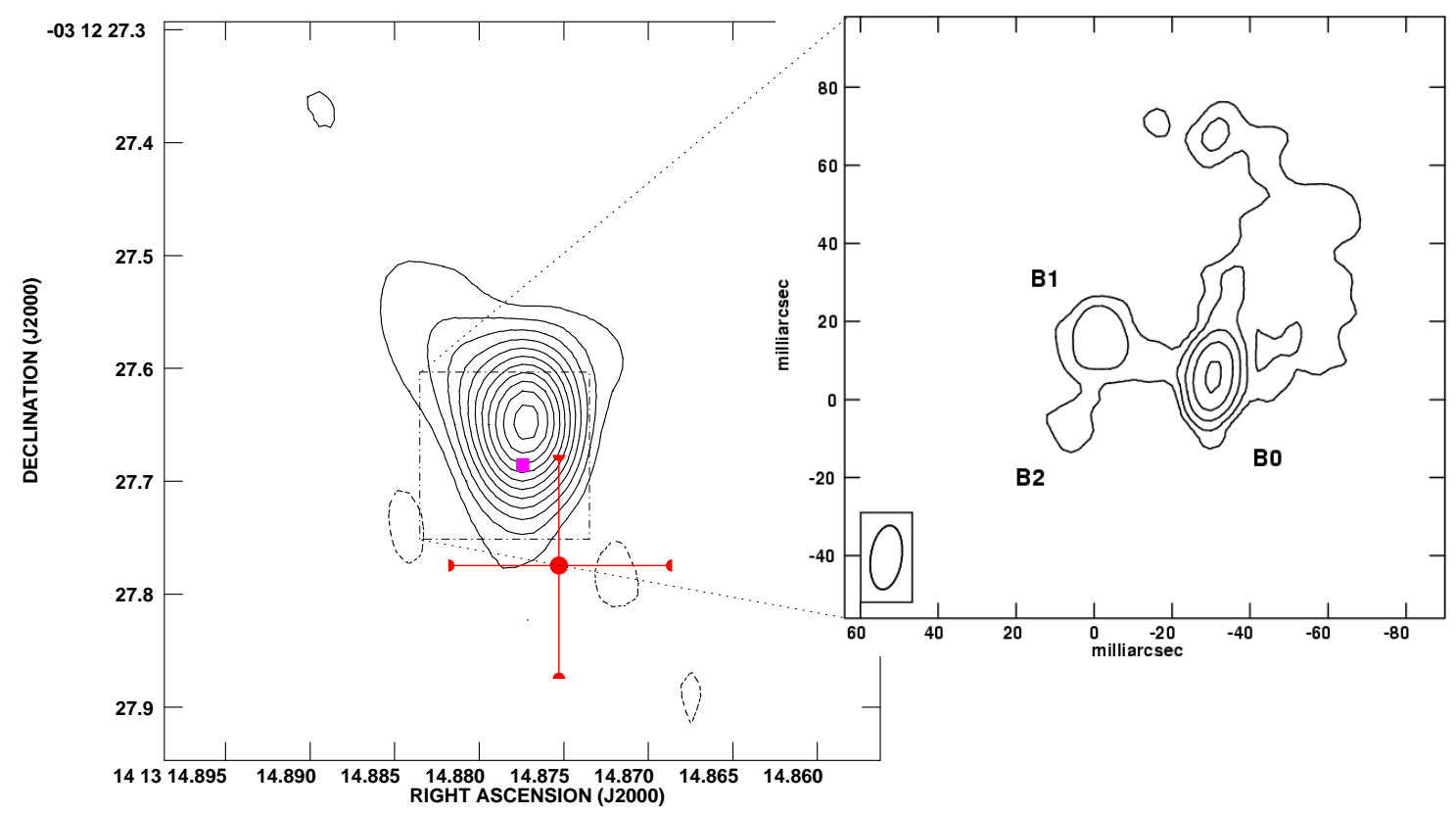

Figure 1: Left panel: VLA A-array K-band continuum image of the nuclear region of NGC 5506. Contour levels are $-1,1,2,3,45678910112.1 \mathrm{mJy}^{-1}$ beam $^{-1}\left(1 \sigma \mathrm{rms}=0.7 \mathrm{mJy} \mathrm{beam}^{-1}\right)$. We show the position of the water maser emission (red circle) in our VLA A-array map (the size of the bars indicates the uncertainty in the position). The dotted-dashed square indicates the area covered by the VLBI map of [13] and the magenta square the position of the brightest source, B0, in this map. Right panel: the EVN and MERLIN 6 $\mathrm{cm}$ image of the nuclear region of NGC 5506 by [13]. Offsets are relative to RAJ2000 $=14^{\mathrm{h}} 13^{\mathrm{m}} 14 .{ }^{\mathrm{s}} 87953$, $\operatorname{Dec}_{\mathrm{J} 2000}=-03^{\circ} 12^{\prime} 27^{\prime \prime} 6799$.

a nuclear jet/wind associated with the brightest radio continuum knot. This latter option seems the most favourite one given the absence of high-velocity satellite maser features (typically present in disk-masers) and being also supported by recent evidence in NGC 4051 of a weak, relatively extended ( $60 \mathrm{pc}$ ) jet ([9], [10], and [12]) and of a shocked outflow ( [15] and [18]).

NGC 5506: the nuclear radio emission of NGC 5506 in our VLA radio continuum K-band image is constituted by an unresolved nuclear source. At higher resolution, in the EVN+MERLIN C-band map of [13], the radio emission is resolved in three luminous radio knots (Fig. 1, right panel), that are described by [13] according to two main scenarios: i) because of its flat spectrum, compactness, and high brightness temperature $\left(\sim 3.6 \times 10^{8} \mathrm{~K}\right)$, the strongest knot (labeled B0) is the putative AGN core; ii) knots B0 and B1 (the second brightest knot) may represent the two components where double-sided jets from the AGN impact the ISM, and hence, according to this picture, the AGN would lie instead between B0 and B1. The third knot (labeled B2) could be low-surface brightness emission extending from the hot spots. The maser emission detected in our VLA DnC and A-array maps has a position for the maser coincident, within the uncertainties, with either one of the aforementioned nuclear components (Fig. 1, left panel), thus, indicating an association of the maser emission with an accretion disk, a radio jet, or a nuclear outflow. Both the evidence for a one-sided jet and an outflow are found for this galaxy ([19], and references therein) and the blue-shifted velocities of the maser lines w.r.t. the systemic velocity may favor, also for this galaxy, 
the jet/outflow maser scenario. Furthermore, [7] recently measured, through the profile of the Xray $\mathrm{FeK} \alpha$ emission line, the inclination of the nuclear disk in NGC5506 to be $i=40^{\circ}$. According to standard amplification models for disk-maser, such an inclination of the disk would be less favourable to produce detectable maser emission.

\section{References}

[1] J. A. Braatz et al.: A Survey for $\mathrm{H}_{2} \mathrm{O}$ Megamasers in Active Galactic Nuclei. II. A Comparison of Detected and Undetected Galaxies, ApJS 110 (1997) 321

[2] J. A. Braatz et al. :A Green Bank Telescope Search for Water Masers in Nearby Active Galactic Nuclei, ApJ 617 (2004) L29

[3] J. A. Braatz et al.: The Megamaser Cosmology Project. II. The Angular-diameter Distance to UGC 3789, ApJ 718 (2010) 657

[4] J. F. Gallimore et al.: The Nature of the Nuclear $\mathrm{H}_{2} \mathrm{O}$ Masers of NGC 1068: Reverberation and Evidence for a Rotating Disk Geometry, ApJ 556 (2001) 694

[5] M. Giroletti \& F. Panessa: The Faintest Seyfert Radio Cores Revealed by VLBI, ApJ 706 (2009) 260

[6] L. J. Greenhill et al.: A Warped Accretion Disk and Wide-Angle Outflow in the Inner Parsec of the Circinus Galaxy, ApJ 590 (2003) 162

[7] M. Guainazzi et al.: Final verdict from XMM-Newton: the X-ray obscured Seyfert galaxy NGC 5506 has a broad Fe Ko line, MNRAS 406 (2010) 2013

[8] Y. Hagiwara: Low-Luminosity Extragalactic Water Masers toward M 82, M 51, and NGC 4051, AJ 133 (2007) 1176

[9] S. Jones et al.: Radio and X-ray variability in the Seyfert galaxy NGC 4051, MNRAS 412 (2011) 2641

[10] A. L. King et al.: A Distinctive Disk-Jet Coupling in the Seyfert-1 Active Galactic Nucleus NGC 4051, ApJ 729 (2011) 19

[11] S. Komossa et al.: Narrow-line Seyfert 1 Galaxies, RMxAC 32 (2008) 86

[12] D. Maitra et al.: A Jet Model for the Broadband Spectrum of the Seyfert-1 Galaxy NGC 4051, ApJ, accepted for publication, [arXiv:1104.5006]

[13] E. Middelberg et al.: Motion and properties of nuclear radio components in Seyfert galaxies seen with VLBI, A\&A 417 (2004) 925

[14] A. B. Peck et al.: The Flaring $\mathrm{H}_{2} \mathrm{O}$ Megamaser and Compact Radio Source in Markarian 348, ApJ 590 (2003) 149

[15] K. A. Pounds \& Vaughan: An extended XMM-Newton observation of the Seyfert galaxy NGC 4051 - I. Evidence for a shocked outflow, MNRAS 413 (2011) 1251

[16] M. Ramolla et al.: Megamaser detection and nuclear obscuration in Seyfert galaxies, A\&A 530 (2011) 147

[17] S. Sawada-Satoh et al.: Positional Coincidence of $\mathrm{H}_{2} \mathrm{O}$ Maser and a Plasma-Obscuring Torus in Radio Galaxy NGC 1052, ApJ 680 (2008) 191

[18] S. Vaughan et al.: The rapid X-ray variability of NGC 4051, MNRAS 413 (2011) 2489

[19] E. Xanthopoulos et al.: Linear radio structures in selected Seyfert galaxies, MNRAS 404 (2010) 1966 